\title{
Multi-query Optimization for Sensor Networks ${ }^{\star}$
}

\author{
Niki Trigoni ${ }^{1}$, Yong $\mathrm{Yao}^{2}$, Alan Demers ${ }^{2}$, Johannes Gehrke ${ }^{2}$, and Rajmohan \\ Rajaraman $^{3}$ \\ 1 Department of Computer Science and Inf. Systems, Birkbeck College, University of London \\ 2 Department of Computer Science, Cornell University \\ 3 College of Computer and Information Science, Northeastern University
}

\begin{abstract}
The widespread dissemination of small-scale sensor nodes has sparked interest in a powerful new database abstraction for sensor networks: Clients "program" the sensors through queries in a high-level declarative language permitting the system to perform the low-level optimizations necessary for energy-efficient query processing. In this paper we consider multi-query optimization for aggregate queries on sensor networks. We develop a set of distributed algorithms for processing multiple queries that incur minimum communication while observing the computational limitations of the sensor nodes. Our algorithms support incremental changes to the set of active queries and allow for local repairs to routes in response to node failures. A thorough experimental analysis shows that our approach results in significant energy savings, compared to previous work.
\end{abstract}

\section{Introduction}

Wireless sensor networks consisting of small nodes with sensing, computation and communication capabilities will soon be ubiquitous. Such networks have resource constraints on communication, computation, and energy consumption. First, the bandwidth of wireless links connecting sensor nodes is usually limited, on the order of a few hundred Kbps, and the wireless network that connects the sensors provides only limited quality of service, with variable latency and dropped packets. Second, sensor nodes have limited computing power and memory sizes that restrict the types of data processing algorithms that can be deployed. Third, wireless sensors have limited supply of energy, and thus energy conservation is a major system design consideration. Recently, a database approach to programming sensor networks has gained interest [1-7], where the sensors are programmed through declarative queries in a variant of SQL. Since energy is a highly valuable resource and communication consumes most of the available power of a sensor network, recent research has focused on devising query processing strategies that reduce the amount of data propagated in the network.

Our Model and Assumptions. We assume that nodes are stationary and battery-powered, and thus severely energy constrained. Users inject queries into a special type of node, referred to as a gateway. The sensor network is programmed through declarative queries posed in a variant of SQL or an event-based language [1-5]. We concentrate on aggregation queries, and the sensor network performs in-network aggregation while routing data from source sensors through intermediate nodes to the gateway.

* This work was partially supported by NSF grants CCR-9983901 and IIS-0330201. 
Existing work has focussed on the execution of a single long-running aggregation query. In our new usage model, we allow multiple users to pose both long-running and snapshot queries (i.e. queries executed once). As new queries occur, they are not sent immediately to the network for evaluation, but are gathered at the gateway node into batches and are dispatched for evaluation once every epoch. The query optimizer groups together queries with the same aggregate operator and optimizes each group separately. Hence, in our presentation of our optimization techniques, we assume that queries use the same aggregate operator. Each epoch consists of a query preparation $(\mathrm{QP})$ and a result propagation $(\mathrm{RP})$ phase. In the $\mathrm{QP}$ phase, all queries gathered during the previous epoch are sent to the network together for evaluation. In the RP phase, query answers are forwarded back to the gateway.

Our model is general enough to include queries with different result frequencies and different lifespans. Although the algorithms proposed in this paper apply to this general usage model, for ease of presentation we will restrict our discussion to a simpler scenario: all queries asked in a QP phase have the same result frequency, and their computation spans a single (and entire) RP phase. The duration of an RP phase is a tunable application-specific parameter. Typically, an RP phase includes multiple rounds of query results. To summarize, an epoch has a QP and an RP phase, and an RP phase has many rounds in which query results are returned to the gateway.

The Intelligent National Park. To give an example of a scenario with multiple queries, consider a sensor network deployed in a national park. Visitors of the park are provided with mobile devices that allow them to access a variety of information about the surrounding habitat by issuing queries to the network through a special purpose gateway. For instance, visitors may wish to know counts of certain animal species in different regions of the park. The region boundaries will vary depending on the location of the visitors. The queries also change with time, as visitors move to different sections of the park, and certain queries are more popular than others. In addition, the sensor readings change probabilistically as animals move around the park, and there might be different update rates during the day than at night.

Our Contributions. This paper addresses the problem of processing multiple aggregate queries in large sensor networks, and makes the following contributions:

- Multi-Query Optimization In Sensor Networks: Concepts and Complexity. We formally introduce the concept of result sharing for efficient processing of multiple aggregate queries. We also address the problem of irregular sensor updates by developing result encoding techniques that send only a minimum amount of data that is sufficient to evaluate the updated queries. Our result sharing and encoding techniques achieve optimal communication cost for sum and related queries (such as count and $a v g$ ). While some of our techniques also extend to $\max$ and $\min$ queries, we show that the problem of minimizing communication cost is NP-hard for these queries [8].

- Distributed Deployment of Multi-Query Optimization. We refine our multi-query optimization algorithms to account for computational and memory limitations of sensor nodes, and present fully distributed implementations of our algorithms. Besides a communication-optimal algorithm, we propose a near-optimal algorithm that significantly decreases the computational effort. We show how to tune our algorithms to take into account the node computational capabilities, and the relative energy ex- 
pended for communication and for computation. In [8], we show how to adapt our algorithms to link failures that change the structure of the dissemination tree.

- Implementation Results Validating our Techniques. We present results from an empirical study of our multi-query optimization techniques with several synthetic data sets and realistic multi-query workloads. Our results clearly demonstrate the benefits of effective result sharing and result encoding. We also present a prototype implementation on real sensor nodes and demonstrate the time and memory requirements of running our code with different query workloads.

Relationship to Traditional Approaches for Multi-Query Optimization (MQO). The problem considered in this paper is significantly different from the traditional MQO problems. The difficulty in devising efficient MQO algorithms for sensor networks is not only in finding common subexpressions, but in dealing with the challenges of distribution and resource constraints at the nodes. This paper is, to the best of our knowledge, the first piece of work to i) formulate this important problem, and ii) give efficient algorithms with provable performance guarantees that are shown to work well in practice.

\section{Optimization Problems and Complexity}

We now formally present the multi-query optimization, and study its complexity, focusing on algorithms that aim to minimize the communication cost of query evaluation ignoring any computation limitations or issues of distributed implementation. In Sect. 3 we will develop fully distributed algorithms that take into consideration the computation and memory constraints in sensor networks.

We consider a set of aggregate queries $Q=\left\{q_{1}, \ldots, q_{m}\right\}$ over a set of $k$ distinct sensor data sources. A set of sensor readings is a vector $x=\left\langle x_{1}, \ldots, x_{k}\right\rangle \in \Re^{k}$. Each query $q_{i}$ requests an aggregate value of some subset of the data sources at some desired frequency. This allows each query $q_{i}$ to be expressed as a $k$-bit vector: element $j$ of the vector is 1 if $x_{j}$ contributes to the value of $q_{i}$, and 0 otherwise. The value of query $q_{i}$ on sensor readings $x$ is expressed as the dot product $q_{i} \cdot x$.

In our multi-query optimization problem, we are given a dissemination tree connecting the $k$ sensor nodes and the gateway, over which the aggregations are executed. Note that our solutions apply to any given tree. The goal is to devise an execution plan for evaluating queries, that minimizes total communication cost. The communication cost includes the cost of query propagation in the QP phase and the cost of result propagation in each round of the RP phase. While we discuss the implementation of the QP phase in detail in Sect. 3.2, we ignore the query propagation cost in the following analysis, since it is negligible compared to the total result propagation costs, whenever the RP phase of an epoch consists of a sufficiently large number of rounds. We consider two classes of aggregation: (i) min queries and (ii) sum queries. Clearly our results for min queries also apply to max, and our results for sum queries can be extended to count, average, moments and linear combinations in the usual way. For min queries, we establish the NP-hardness of the multi-query optimization problem using a straightforrwad reduction from the Set Basis problem [8].

Complexity of sum Queries: For sum queries the underlying mathematical structure is a field. We can exploit this fact, using techniques from linear algebra to optimize the 
number of data values that must be communicated. Let $N$ be an arbitrary node in the tree. Let $P(N)$ denote the parent of $N$ and let $T_{N}$ denote the subtree rooted at $N$. We denote as $x(N)$ the vector of sensor values in the subtree $T_{N}$ and $Q_{x(N)}$ the set of query vectors projected only onto sensors in $T_{N}$.

We present a simple method to minimize the amount of data that $N$ sends to $P(N)$ in each round. Let $B\left(Q_{x(N)}\right)=\left\{b_{1}, \ldots, b_{n}\right\}$ be a basis of the subspace of $\Re^{k}$ spanned by $Q_{x(N)}$. Then any query $q \in Q_{x(N)}$ can be expressed as a linear combination of the basis vectors $q=\sum_{j} \alpha_{j} \cdot b_{j}$, where $\alpha_{j} \in \Re, j=1, \ldots, n$. By linearity of inner product we get, for sensors $x(N)$ (in the subtree $T_{N}$ )

$$
q \cdot x(N)=\left(\sum_{j} \alpha_{j} \cdot b_{j}\right) \cdot x(N)=\sum_{j} \alpha_{j} \cdot\left(b_{j} \cdot x(N)\right)
$$

That is, to evaluate the answers of queries in $Q_{x(N)}$ it suffices to know the answers for any basis of the query space spanned by $Q_{x(N)}$. Any maximal linearly independent subset of $Q_{x(N)}$ is a (not necessarily orthogonal or normal) basis of the space and every such basis has the same cardinality. So we can use any maximal linearly independent subset of $Q_{x(N)}$ as our basis, and $N$ can forward the answers of the queries in this basis to $P(N)$. The parent $P(N)$, using the same set of basis vectors, can easily interpret the reduced results that it receives from $N$. We assume that $N$ and $P(N)$ use the same algorithm in order to identify the basis vectors of $Q_{x(N)}$, and the factors $\alpha_{j}$. We refer to this procedure as linear reduction.

Theorem 1. The size of the query result message sent by the above algorithm in each round is optimal.

\section{Multi-Query Optimization}

The linear reduction technique outlined in Sect. 2 provides an elegant solution for minimizing the cost of processing multiple sum queries. However, a number of system considerations have to be taken into account to apply to a real sensor network. In this section, we develop fully distributed multi-query optimization algorithms for sum queries. Due to space constraints, we do not consider the impact of failures on our algorithms. We refer the reader to the full paper for a discussion on failures and detailed experiments that measure the tradeoff between communication and computation cost in the presence of failures [8]. We start our discussion by introducing the notion of equivalence class, which is central to the algorithms proposed in the remainder of the section.

\subsection{Queries and equivalence classes}

Rectangular Queries: We have represented each query as a $k$-bit vector, where $k$ is the number of sensors. Expressing queries in this form requires that the user have complete knowledge of the sensor topology. It is more natural, and generally more compact, to represent queries spatially. We focus our attention on queries that aggregate sensor values within a rectangular region, and represent such a query as a pair of points $\left(\left(x_{0}, y_{0}\right),\left(x_{1}, y_{1}\right)\right)$ at opposite corners of the rectangle. Since queries do no longer enumerate nodes specifically, we can even evaluate queries in an acquisitional manner [9], e.g. by selecting a sample of sensor values generated within a query rectangle. 


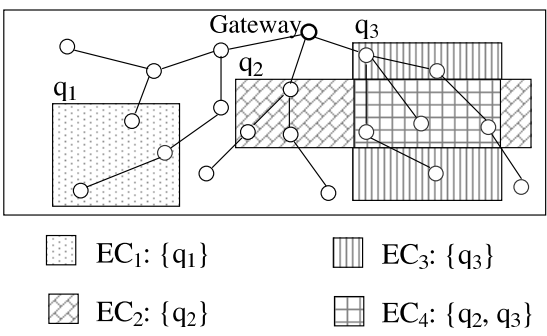

Fig. 1. Equivalence classes formed by three queries.

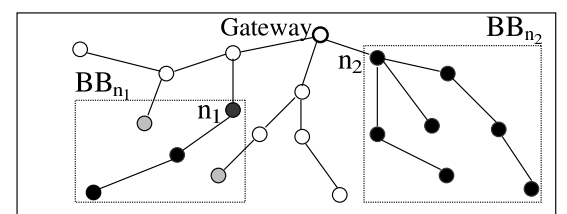

Fig. 2. The bounding box of a subtree is the minimum rectangle that covers all sensors in the subtree. Grey nodes represent sensors that belong to a bounding box of a subtree without belonging to the subtree.

Equivalence Classes (ECs): To deal efficiently with rectangular queries and distribution, we introduce the notion of Equivalence Class $(E C)$. An equivalence class is the union of all regions covered by the same set of queries. For example, Fig. 1 shows that queries $\left\{q_{1}, q_{2}, q_{3}\right\}$ form four $\mathrm{ECs}\left\{E C_{1}, E C_{2}, E C_{3}, E C_{4}\right\}$, each one of which corresponds to a different set of queries. For instance, $E C_{4}$ is covered only by queries $q_{2}$ and $q_{3}$, and can be represented by the column bit-vector $[0,1,1]^{T} ; E C_{1}$ is represented by $[1,0,0]^{T}, E C_{2}$ by $[0,1,0]^{T}$ and $E C_{3}$ by $[0,0,1]^{T}$. Notice that an equivalence class is not necessarily a connected region (see $E C_{2}$ ). An equivalence class may contain no sensors. Equivalence classes are identified based solely on spatial query information; they are independent of the node locations in the network or of the dissemination tree that connects nodes. We can, however, speak of the value of an equivalence class - this is the aggregate of the data values of sensors located in the EC region. The value of an EC can be obtained by a subset of sensors located in the EC region, if an acquisitional processing style is adopted.

Query Vectors and Query Values: We can now express queries in terms of ECs as follows. We number equivalence classes (instead of sensors) from 1 to $\ell$, where $\ell$ is the number of equivalence classes. Let $x$ denote the column vector in $\Re^{\ell}$ representing the values of the equivalence classes; thus, $x_{i}$ denotes the sum of (all or a sample of) sensor values in $E C_{i}$. Each query $q$ is a linear combination of the set of equivalence classes and can be captured by a row vector in $\{0,1\}^{\ell}$. For example, query $q_{3}$ in Fig. 1 can be represented as the vector $[0,0,1,1]$, since it only covers $E C_{3}$ and $E C_{4}$. The value of a query $q$ given the EC values $x$ is simply the product $q \cdot x$. Given the above representation of the queries and EC values, it is natural to represent a set of $m$ queries as an $m \times \ell$ (bit) matrix $Q$, in which the $(i, j)$ element is 1 if the $i$ th query in $Q$ covers the $j$ th equivalence class. The value of the query set $Q$ given the EC values $x$ is again given by the product $Q \cdot x$, which is a column vector in $\Re^{m}$. We often refer to the rows of a query-EC matrix as query vectors, and to the columns as EC vectors.

Bounding Boxes (BBs): Expressing queries in terms of ECs brings out the dependencies among queries. In order to exploit these dependencies fully, each node needs to view queries in the context of its subtree, rather than the entire network. Therefore, in our algorithms, a node expresses queries in terms of ECs intersecting with its subtree; an EC intersects with a subtree if any of the sensor nodes in the subtree lies within the EC region. A node $N$ can accurately determine which ECs intersect with subtree $T_{N}$, 
if it either knows the locations of all nodes in $T_{N}$ or receives from its children a list of all ECs intersecting with their subtrees. Both approaches are prohibitive in terms of the communication involved. An approximation of the set of equivalence classes intersecting $T_{N}$ can be obtained if we consider the minimum rectangle that contains all sensors in the subtree. This rectangle is hereafter called the bounding box of $T_{N}$ and is denoted as $B B_{N}$. Figure 2 depicts the bounding boxes of the subtrees rooted at nodes $n_{1}$ and $n_{2}$. Note that a bounding box may contain nodes that do not belong in the subtree (grey nodes in Fig. 2).

Queries and ECs projected to the bounding box of a subtree: Let $X_{N}$ denote the set of equivalence classes that intersect with the bounding box of the subtree $T_{N}$. It is easy to see that $X_{N}$ is a superset of the equivalence classes that actually intersect with the subtree $T_{N}$. For given query set $Q$, we let $Q_{N}$ denote the projection of $Q$ on to $X_{N}$; that is, we obtain $Q_{N}$ by setting all entries of $Q$ that appear in columns not in $X_{N}$ to be zero. Duplicate and zero rows are removed. We extend the notation to let $x_{N}$ denote the vector of projected EC values onto the subtree $T_{N}$ (not onto $B B_{N}$ since a node $N$ can only receive values generated by its descendants). The $i$ th entry corresponds to the sum of sensor values lying in the intersection of $E C_{i}$ and the subtree $T_{N}$. The entries of ECs that do not intersect with $T_{N}$ are set to 0 . If we denote the values of queries $Q$ that are contributed from sensors in the subtree $T_{N}$ as $V(Q, N)$, then the vector of values of $Q_{N}$ contributed from subtree $T_{N}$ is $V\left(Q_{N}, N\right)=Q_{N} \cdot x_{N}$.

\subsection{The Query Preparation (QP) phase}

The query preparation phase consists of three steps: a bounding-box calculation step, a query propagation step, and an EC evaluation step. Some of our algorithms for the RP phase do not require the evaluation of ECs, in which case the last step is omitted.

Bounding-box calculation: A dissemination tree is first created using a simple flooding algorithm. Given the dissemination tree, each node $N$ computes the bounding box $B B_{N}$ of its subtree $T_{N}$ from the bounding boxes of the subtrees of its children (if any) as follows. If $x$, (resp., $\left.x^{\prime}\right)$ and $y$ (resp, $y^{\prime}$ ) are the smallest (resp., largest) $x$ - and $y$-coordinates of the child bounding boxes, then $(x, y)$ and $\left(x^{\prime}, y^{\prime}\right)$ form two opposite corner points of the bounding box of $N$.

Query propagation: The next step is to send query information down the dissemination tree. We distinguish query propagation schemes based on whether bounding boxes are used to reduce the query propagation cost: (i) AllQueries: flood all queries to the entire network; (ii) BBQueries: each node propagates down only queries that have a non-empty intersection with its bounding box. This is performed using semantic routing information, discussed in detail in [9]. Once a node receives query information, it computes for each round in the epoch the set of queries that are active in the round.

EC computation: Given a set of $m$ query rectangles, we can compute all the ECs formed by the $m$ queries using a two-dimensional sweep algorithm in $O\left(m^{3}\right)$ time using $O\left(\mathrm{~m}^{3}\right)$ space. Due to space constraints, we defer the algorithm description and its analysis to the full paper. Using this algorithm, each node locally computes the ECs intersecting with its bounding box. 


\subsection{The Result Propagation (RP) phase}

Each RP phase consists of a number of rounds, in which aggregation results are forwarded through the tree paths from the leaves to the gateway. Consider a result message sent by a node $N$ to its parent $P(N)$. The forwarded data should be sufficient to evaluate $V\left(Q_{N}, N\right)$, i.e. the contribution of sensors in $T_{N}$ to the values of the projected queries $Q_{N}$. A result message consists of a pair 〈RESUltCode, RESUltDatA); RESULTDATA includes updated values, and RESULTCODE encodes what has been updated, showing how to interpret the values in RESULTDATA.

We now propose a series of result propagation algorithms, all of which use the above message format. These algorithms can be classified according to four dimensions. The first two dimensions are the methods employed for computing the RESULTCODE and RESUltDATA components. The third dimension is whether the linear reduction technique of Sect. 2 is applied. The last dimension is whether these choices are identical for all nodes, yielding a pure algorithm, or these choices may differ across nodes, yielding a hybrid algorithm.

Pure algorithms without reduction: We consider two methods for determining the RESULTCODE component of a result message. In Query-encoding, a node sends to its parent information about which queries have been updated since the last round. Formally, let $U$ pdRows $\left(Q_{N}\right)$ be the matrix derived from $Q_{N}$ after removing all queries (row vectors) that are not affected by the current sensor updates in $T_{N}$. Both $N$ and $P(N)$ agree on unique labels for the queries in $Q_{N}$ from the integer interval $\left[1,\left|Q_{N}\right|\right]$. Then, RESULtCODE consists of a set of $\lg \left|Q_{N}\right|$-bit labels listing the queries in $\operatorname{UpdRows}\left(Q_{N}\right)$. We note that Query-encoding does not require computation of equivalence classes. In EC-encoding, a node sends to its parent information about which equivalence classes have been updated since the last round. Let $\operatorname{UpdCols}\left(Q_{N}\right)$ be the matrix derived from $Q_{N}$ after removing all ECs (column vectors) that do not include any updated sensors in $T_{N}$ (and after removing duplicate and zero rows). Since both $N$ and $P(N)$ can compute $X_{N}$ (i.e. the set of equivalence classes that intersect with $\left.B B_{N}\right)$ they can agree on a unique label in the range $\left[1,\left|X_{N}\right|\right]$ for each equivalence class in $X_{N}$. In EC-encoding, RESULTCODE includes the identifiers of ECs (columns) of $\operatorname{UpdCols}\left(Q_{N}\right)$.

We also consider two methods for populating the RESULTDATA component of a result message that a node sends to its parent. In the Query-data approach, RESULTDATA is the set of values of updated queries. In the $E C$-data approach, RESULTDATA is the set of values of updated EC values.

One can combine the two dimensions above to obtain four different algorithms for the RP phase: QueryQuery, QueryEC, ECQuery and ECEC, respectively, where the first part of the name refers to the encoding, and the second part to the data. EC-encoding results in messages with smaller RESULTDATA components than Query-encoding, independent of whether the Query-data or EC-data policy is used. This is because both (row and column) dimensions of $\operatorname{UpdCols}\left(Q_{N}\right)$ are smaller than those of $\operatorname{UpdRows}\left(Q_{N}\right)$. Therefore, if the computational capabilities of the sensor nodes allow EC-computations, then we only consider ECQuery and ECEC. On the other hand, if the computational limitations of the sensor nodes do not allow them to compute the ECs, then QueryQuery 
is the only algorithm of interest. Consequently, we focus our attention on three of these four algorithms, namely, QueryQuery, ECQuery, and ECEC.

- ECQuery: In the RESUltCodE component, each node $N$ sends to $P(N)$ the identifiers of the updated ECs in the subtree rooted at $N$. In the RESULTDATA component, node $N$ includes delta values only of the distinct row vectors of matrix $\operatorname{UpdCols}\left(Q_{N}\right)$. That is, query vectors are projected only onto the updated ECs (columns), and one value is sent for each distinct projected query vector.

- QueryQuery: In the RESULTCODE component of the message that $N$ sends to $P(N)$, it includes the identifiers of updated queries. In RESULTDATA, node $N$ includes delta values of the distinct row vectors of matrix $\operatorname{UpdRows}\left(Q_{N}\right)$. Since the number of distinct query (row) vectors in $\operatorname{UpdRows}\left(Q_{N}\right)$ is larger or equal to their number in $U p d \operatorname{Cols}\left(Q_{N}\right)$, the size of RESULTDATA in QueryQuery is larger or equal to its counterpart in ECQuery.

- ECEC: The RESUlTCODE here is identical to that of ECQuery. Unlike ECQuery, ECEC sends up EC values in the RESULTDATA component of the message. For each updated EC in the subtree, it sends up the aggregate value of all sensors in the intersection of the EC and the subtree $T_{N}$.

An optimal pure algorithm using linear reduction: Both ECQuery and ECEC decrease the communication cost of result propagation by explicitly encoding irregular updates. Additional communication savings can be achieved by carefully applying the linear reduction technique (introduced in Sect. 2) in a distributed manner to reduce the size of propagated irregular updates. We now present the algorithm ECReduced which uses EC-encoding, and is provably optimal with respect to the amount of result data that is communicated. The RP phase of ECReduced at each node consists of two steps: i) a basis evaluation step and ii) a result evaluation step. Detailed pseudocode for both steps is presented in [8]. The basis evaluation step is executed whenever the set of active queries changes or the set of updated ECs changes. Thus, if every query has the same frequency and all sensors are updated regularly (D-scenario), then the basis evaluation step is executed only once at the beginning of the RP phase. This step is the most computationally demanding part of our algorithm since it involves matrix linear reduction; the complexity of reducing a matrix with $m$ rows and $n$ columns is $O\left(m n^{2}\right)$.

Basis evaluation step: Consider a node $N$ with $c h$ children nodes. Node $N$ initially performs $c h$ row-based linear reductions on matrices $\operatorname{UpdCols}\left(Q_{N_{k}}\right), k=1, \ldots, c h$, in order to interpret the results received from its children $N_{1}, \ldots, N_{c h}$. It derives a coefficient matrix $A_{N_{k}}$ for each child $k$, such that the product of $A_{N_{k}}$ and the basis vectors $B\left(U p d \operatorname{Cols}\left(Q_{N_{k}}\right)\right)$ yields the original projected queries $\operatorname{UpdCols}\left(Q_{N_{k}}\right)$. Node $N$ then reduces its own query-EC matrix $\operatorname{UpdCols}\left(Q_{N}\right)$ into a set of linearly independent query vectors. Overall, $N$ performs $c h+1$ matrix reductions.

Result evaluation step: This step is executed once per round of the RP phase, and it includes simple operations wrt time and memory space. Relying on the output of the basis evaluation step, node $N$ combines the incoming (delta) values received from its children and forwards a minimum number of values to its parent $P(N)$. Details of this step are given in [8]. In summary, for each child $N_{k}$, node $N$ evaluates the values of queries (row vectors of) $\operatorname{UpdCols}\left(Q_{N_{k}}\right)$, based on the values of the basis vectors (received from child $k$ ) and matrix $A_{N_{k}}$ (from previous step). Combining the values of 
$\operatorname{UpdCols}\left(Q_{N_{k}}\right)$ (and the node's own sensor value), node $N$ proceeds to evaluate the results of queries $\operatorname{UpdCols}\left(Q_{N}\right)$ for the entire subtree $T_{N}$. It is sufficient to evaluate only the values of queries that belong to the basis $B\left(\operatorname{UpdCols}\left(Q_{N}\right)\right)$. Only those values are finally forwarded to the parent node $P(N)$. Notice that if all nodes use the same algorithm to linearly reduce a query matrix, there is no need to communicate the selected basis vectors; a node only forwards up the values of these vectors to its parent. The following result is derived from Theorem 1.

Theorem 2. The size of the RESULTDATA component in the ECReduced algorithm is optimal; it is a lower bound on the size of the optimal result message.

Hybrid algorithms with no reduction: The algorithms introduced so far are executed in an identical manner at all nodes. We now consider two hybrid algorithms that perform differently across nodes, depending on the load of results contributed by the underlying subtrees. The first algorithm, referred to as HybridBasic, attempts to approximate the optimal cost achieved by the ECReduced algorithm, while avoiding the high computational requirements for linear reduction.

- HybridBasic: Consider the bounding box of a node and the set of queries and ECs intersecting with the bounding box. For a given sensor update rate, when the number of (projected) queries is small, the number of (projected) ECs is greater than the number of queries. In this case, the ECQuery algorithm is expected to outperform the ECEC algorithm. However, for a large number of queries the equivalence classes might be fewer than the queries. In this case, the ECEC algorithm is expected to outperform the ECQuery algorithm. The point where the two algorithms cross depends on the sensor update frequency. The HybridBasic algorithm combines the ECEC and ECQuery approaches. A node selects the approach that locally yields the least cost, and sends an additional bit to denote its choice. The only constraint is that if a child uses the ECQuery approach, it only provides information about the values of updated queries; hence, its parent can only implement the ECQuery approach. On the contrary, a parent of a node that implements ECEC can implement either of the two approaches.

Surprisingly, HybridBasic performs extremely well in terms of communication; as will be shown in Sect. 4, it closely approximates the cost of the ECReduced algorithm, without requiring a linear reduction task. In fact, HybridBasic can be viewed as an approximate application of linear reduction in the following sense: the rank of a matrix is always smaller or equal to the smallest dimension of the matrix; given a query-EC matrix, HybridBasic effectively chooses to propagate values of row vectors (queries), or of column vectors (ECs) depending on which ones are fewer. In practice, this policy works well, since the cardinality of the smallest matrix dimension often coincides with the matrix rank.

HybridBasic assumes that each node is able to evaluate equivalence classes within the bounding box of its subtree. The following algorithm, named HybridWithThreshold, lifts this requirement for nodes close to the gateway, whose bounding boxes overlap with many queries.

- HybridWithThreshold: If the input query workload is light, EC evaluation for the entire network is easy to perform locally at each node. Otherwise, nodes close to the 
leaves may opt for local EC computation, i.e. computation of ECs within the context of the bounding box of the node's subtree. As we approach the gateway, the bounding box of a node's subtree increases, and so do the number of query rectangles that intersect with the bounding box. The computational cost of evaluating ECs may become prohibitively expensive for nodes close to the gateway. The HybridWithThreshold algorithm behaves like the HybridBasic algorithm at nodes that are able to perform EC computation. When the effort for EC computation exceeds a certain threshold at a node (its computational capability), the node switches to Query-encoding and sends up one result per updated query.

\section{Experimental Evaluation}

In this section we measure the communication cost of the proposed algorithms using a home-grown simulator. We also present our feasibility test of the linear reduction technique, which we performed on the Mica2 mote. In the full version of our paper [8] we evaluate how the proposed algorithms trade communication for computation; we also show the benefits of our techniques by drawing data from a real sensor network infrastructure deployed in the Intel Berkeley Research Lab.

\subsection{Synthetic experimental setup}

We deploy 400 sensors in a square region of $400 \mathrm{~m}^{2}$ and randomly select their $x$ and $y$ coordinates to be any real numbers in $[0,20]$. We ensure that with a communication range of $2 m$ the random deployment of nodes results in a (100\%) connected network (otherwise the random deployment is repeated). A flooding algorithm is used to generate a minimum spanning tree that connects all nodes to the gateway. Each node selects as its parent a randomly chosen neighbor that lies on a shortest path to the root. The queries considered in our framework are sum queries that cover all sensors in a rectangular area. In our experiments we test a number of different query workloads, each defined as a set of tuples of the form (numberOfQueries, minQueryWidth, maxQueryWidth, minQueryHeight, maxQueryHeight). We assume that all the queries in a workload have the same frequency. We set the minimum values of the query dimensions (minQueryWidth, minQueryHeight) to $1 \mathrm{~m}$ and the maximum values to $20 \mathrm{~m}$. Given query input patterns, a random workload generator generates specific instances in each epoch that satisfy the patterns. The sensor update workload defines the probability that a sensor is updated at the end of a round. Given a sensor update input pattern, a random workload generator selects a specific set of sensors to be updated in a round.

For simplicity, we assume long-running queries that are propagated once at the beginning of an epoch (in the QP phase) and are evaluated at every round of the RP phase until the end of the epoch. Since the query propagation cost occurs once per epoch, it is negligible compared to the result propagation cost and is not accounted for. In our evaluation, we measure the result (communication) cost per round, averaged over 200 rounds (10 epochs of 20 rounds each). 

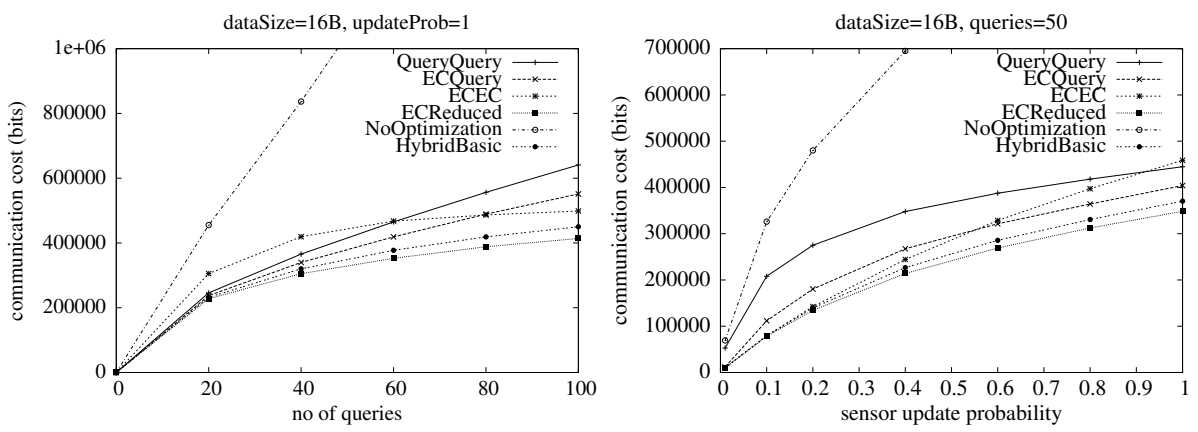

Fig. 3. Comm. cost of algorithms (D-scenario). Fig. 4. Comm. cost of algorithms (I-scenario).

\subsection{Communication cost}

To make the measurements of communication cost realistic, we consider a packet size of 34 bytes (similar to the size of TOS_Msg used for Mica motes) that consists of a 5 -byte header and a 29-byte payload. If the number of bits in a message is $x$, then the communication cost is $\lceil x / 29\rceil \times 34$; that is, we account for a fixed header cost ( 5 bytes) and only consider fixed size packets. The size of each query result is set to 16 bytes.

Deterministic sensor updates: In Fig. 3, we compare the performance of different algorithms as we increase the number of queries sent together for evaluation at the beginning of an epoch. In this initial experiment, we assume that all sensors are updated in each round with probability 1 (D-scenario). We first compare our techniques with the existing approach, namely an extension of the TAG algorithm [3] to process multiple queries. Since this algorithm, which we refer to as NoOptimization, performs in-network aggregation independently for each query, the average (per round) result propagation cost increases linearly in the number of queries. The performance advantage of our proposed techniques is apparent even for light query workloads.

Figure 3 validates our analysis of Sect. 3.3 that EC-encoding outperforms Queryencoding, if we restrict our attention to communication cost. Between ECQuery and ECEC, Fig. 3 shows that ECQuery outperforms ECEC for query workloads with less than 80 queries, but as we increase the number of queries, the number of ECs became smaller than the number of queries and ECEC wins.

We now consider the cost and benefit of the reduction technique in the D-scenario. Figure 3 shows that the proposed ECReduced algorithm performs better than all the other algorithms, thus validating Theorem 2 . An interesting observation is that the HybridBasic algorithm performs almost as well as the ECReduced algorithm, without requiring any computational cost for linear reduction (Fig. 3). This shows that a very simple distributed algorithm, which can easily be implemented on constrained sensor nodes, gives a very good approximation of the optimal solution.

In addition to our simulations, we implemented the linear reduction technique on the Berkeley Mica2 motes (4MHz ATMEL processor128kB flash, 4kB RAM, 4kB ROM) using the $\mathrm{Nes} C$ programming language. We measured the time in seconds required for reducing an $m \times m$ matrix of floats as a function of $m$. The observed time grows as 


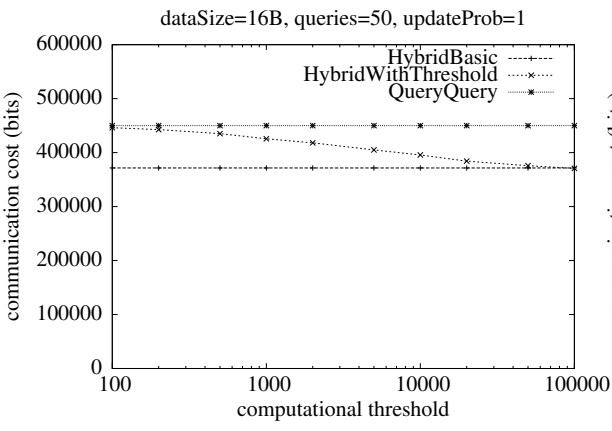

Fig. 5. HybridWithThreshold (D-scenario).

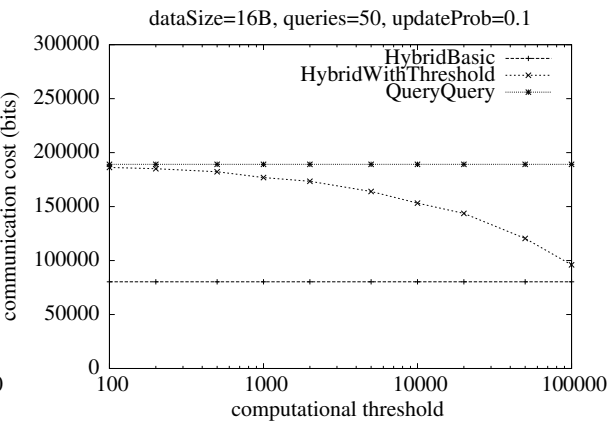

Fig. 6. HybridWithThreshold (I-scenario).

$\Theta\left(m^{3}\right)$, which is consistent with the complexity of the reduction algorithm. The code for matrix reduction was compiled with "make mica" to 12604 bytes in ROM and 428 bytes in RAM. For matrices of dimension from 5 to 15, the linear reduction algorithm takes 0.07 to 1 seconds, but the algorithm time increases rapidly for larger matrices.

Probabilistic sensor updates: In Fig. 4, we compare algorithms as we vary the probability that a sensor generates an updated reading in a given round. We set the number of queries to 50. Recall that in the D-scenario (Fig. 3), which corresponds to the I-scenario with probability 1, ECQuery is preferred to ECEC for workloads of less than 80 queries. As we decrease the sensor update probability to less than 0.6, however, Fig. 4 shows that it becomes beneficial for nodes to send up EC values instead of query values in RESULTDATA. For an update rate of $10 \%$, ECEC is $30 \%$ cheaper than ECQuery. The ECReduced algorithm, which applies the linear reduction technique in a distributed manner, outperforms all other algorithms (Fig. 4). Moreover, the HybridBasic algorithm has a very good performance, approaching closely the cost of ECReduced.

In Figs. 5 and 6, we consider the limited computational power of sensor nodes. From the two algorithms that do not require EC computation (NoOptimization and QueryQuery), we only consider QueryQuery because it has smaller communication cost. Among the algorithms that do not perform reduction but require EC computation, we only consider HybridBasic because it has similar computational cost with the others yet smaller communication cost. We omit ECReduced because it requires matrix reduction without yielding noteworthy cost savings compared to HybridBasic. In Figs. 5 and 6, we set the sensor update probability to 1 and 0.1 respectively. In both figures, QueryQuery has a higher cost than HybridBasic. The former algorithm does not require knowledge of ECs, whereas the latter assumes knowledge of ECs independent of the nodes' computational capabilities. We study the performance of the HybridWithThreshold algorithm, where the significance of the threshold value is as follows: if the effort of computing ECs at a node $N$ (measured as $m^{3}$, where $m$ is the number of distinct projected queries onto the local bounding box $B B_{N}$ ) exceeds the threshold value at $N$, then EC computation cannot be performed, and the node switches to using the QueryQuery algorithm. Figure 5 shows that as we increase the threshold value (plotted on a logarithmic scale), more nodes are able to compute ECs, and the cost of HybridWithThreshold approaches the cost of HybridBasic. 


\section{Related work}

Query processing in sensor networks. Several research groups have focused on innetwork query processing as a means of reducing energy consumption. The TinyDB Project at Berkeley investigates query processing techniques for sensor networks including an implementation of the system on the Berkeley motes and aggregation queries [1-5]. An acquisitional approach to query processing is proposed in [9], in which the frequency and timing of data sampling is discussed. The sensor network project at USC/ISI group $[10,11]$ proposes an energy-efficient aggregation tree using data-centric reinforcement strategies (directed diffusion). A two-tier approach (TTDD) for data dissemination to multiple mobile sinks is discussed in [12]. An approximation algorithm for finding an aggregation tree that simultaneously applies to a large class of aggregation functions is proposed in [13]. Duplicate insensitive skethches for approximate aggregate queries are discussed in $[14,15]$. Our study differs from previous work in that we consider multi-query optimization for sensor networks.

Communication protocols for sensor networks. The data dissemination algorithms that we study in this paper are all aimed at minimizing energy consumption, a primary objective in communication protocols designed for sensor (and ad hoc) networks. A number of MAC and routing protocols have been proposed to reduce energy consumption in sensor networks [16-23] While these studies consider MAC and routing protocols for arbitrary communication patterns, our study focuses on multi-query optimization to minimize the amount of data.

\section{Conclusions and Future Work}

Our work addresses several issues in the area of Sensor Databases. We have introduced two major extensions to the standard model of executing a single long-running query: A workload of multiple aggregate queries and a workload of sensor data updates. We have given efficient algorithms for multi-query optimization, and tested their performance in several scenarios. To the best of our knowledge this is the first work to formally examine the problem of multi-query optimization in sensor networks.

The main conclusions drawn in this paper are the following: First, the notion of equivalence class (EC) is important for distributed query evaluation: encoding sensor updates in terms of ECs enables better compression of the result messages. Second, the result data size is minimized for a certain class of aggregate queries (sum, count and avg) by applying the linear reduction technique in a distributed manner. Third, in applications where the computationally expensive task of linear reduction is infeasible for the nodes, a very good approximation of the optimal can be obtained by having each node select an appropriate local data encoding strategy. This encoding strategy can itself be defined in terms of a threshold that specifies the computational limitation.

There are a number of directions for further research. First, we would like to extend our ideas to a wider class of aggregation functions. Second, our paper has focused on accurate query evaluation. It would be worthwhile to study approximate query processing and obtain error-energy tradeoffs. We would also like to adapt our techniques to multi-path aggregation methods that provide more fault-tolerance. 


\section{References}

1. Hellerstein, J., Hong, W., Madden, S., Stanek, K.: Beyond average: Towards sophisticated sensing with queries. In IPSN. (2003)

2. Madden, S., Franklin, M.: Fjording the stream: An architecture for queries over streaming sensor data. In ICDE. (2002)

3. Madden, S., Franklin, M., Hellerstein, J., Hong, W.: Tag: A tiny aggregation service for ad-hoc sensor networks. In OSDI. (2002)

4. Madden, S., Hellerstein, J.: Distributing queries over low-power wireless sensor networks. In SIGMOD. (2002)

5. Madden, S., Szewczyk, R., Franklin, M., Culler, D.: Supporting aggregate queries over adhoc sensor networks. In WMCSA. (2002)

6. Yao, Y., Gehrke, J.: The Cougar approach to in-network query processing in sensor networks. Sigmod Record 31 (2002)

7. Yao, Y., Gehrke, J.: Query processing in sensor networks. In CIDR. (2003)

8. Trigoni, N., Yao, Y., Demers, A., Gehrke, J., Rajaraman, R.: Multi-query optimization for sensor networks. In TR2005-1989, Cornell Univ. (2005)

9. Madden, S., Franklin, M., Hellerstein, J., Hong, W.: The design of an acquisitional query processor for sensor networks. In SIGMOD. (2003)

10. Heidemann, J., Silva, F., Intanagonwiwat, C., Govindan, R., Estrin, D., Ganesan, D.: Building efficient wireless sensor networks with low-level naming. In SOSP. (2001) 146-159

11. Heidemann, J., Silva, F., Yu, Y., Estrin, D., Haldar, P.: Diffusion filters as a flexible architecture for event notification in wireless sensor networks. In ISI-TR-556, USC/ISI. (2002)

12. Ye, F., Luo, H., Cheng, J., Lu, S., Zhang, L.: A two-tier data dissemination model for largescale wireless sensor networks. In MOBICOM. (2002)

13. Goel, A., Estrin, D.: Simultaneous optimization for concave costs: Single sink aggregation or single source buy-at-bulk. In SODA. (2003)

14. Considine, J., Li, F., Kollios, G., Byers, J.: Approximate aggregation techniques for sensor databases. In ICDE. (2004)

15. Nath, S., Gibbons, P.: Synopsis diffusion for robust aggregation in sensor networks. In SENSYS. (2004)

16. Heinzelman, W., Wendi, R., Kulik, J., Balakrishnan, H.: Adaptive protocols for information dissemination in wireless sensor networks. In MOBICOM. (1999)

17. Johnson, D., Maltz, D.: Dynamic source routing in ad hoc wireless networks. In: Mobile Computing. Volume 353 of The Kluwer International Series in Engineering and Computer Science. (1996)

18. Perkins, C.: Ad hoc on demand distance vector (aodv) routing. (Internet Draft 1999, http://www.ietf.org/internet-drafts/draft-ietf-manet-aodv-04.txt)

19. Perkins, C., Bhagwat, P.: Highly dynamic destination-sequenced distance-vector routing (DSDV) for mobile computers. In SIGCOMM. (1994) 234-244

20. Xu, Y., Bien, S., Mori, Y., Heidemann, J., Estrin, D.: Topology control protocols to conserve energy inwireless ad hoc networks. In TR6, UCLA/CENS (2003)

21. Xu, Y., Heidemann, J., Estrin, D.: Geography-informed energy conservation for ad hoc routing. In MOBICOM. (2001) 70-84

22. Ye, W., Heidemann, J., Estrin, D.: An energy-efficient MAC protocol for wireless sensor networks. In INFOCOM. (2002) 1567-1576

23. Ye, W., Heidemann, J., Estrin, D.: Medium access control with coordinated, adaptive sleeping for wireless sensor networks. In ISI-TR-567, USC/ISI. (2003) 